\title{
The role of modern geodynamic movements in the formation of geomechanical problems in subsoil use
}

\author{
Anatoly Sashourin ${ }^{* 1}$, Andrey Panzhin ${ }^{1}$, July Konovalova ${ }^{1}$ and Vladimir Ruchkin ${ }^{1}$ \\ ${ }^{1}$ Institute of mining of UB RAS, Ekaterinburg, Russia
}

\begin{abstract}
The paper describes the role of geomechanical processes and phenomena in the development and manifestation of hazardous situations in the development of mineral deposits, threatening the working personnel and the surrounding population. The studies of the Ural Scientific School of geomechanics functioning in the Institute of Mining of Ural Branch of RAS have established that the basis for the formation of hazardous geomechanical processes and phenomena are the fundamental properties of the rock mass - hierarchical blocking and constant mobility caused by the recent geodynamical movements. The constant mobility of hierarchical block medium of rock mass under the influence of trend and short-term recent geodynamical movements gives rise to the phenomenon of the secondary structuring. The recent geodynamical movements concentrate at the borders of the secondary structures and result in the deformations exceeding by 2 - 5 times the intrablock and integral deformations and representing the main source of the hazardous impact on the mining objects and on the surrounding population. The technology of the geological environment diagnostics for the forecast of hazardous mining situations is aimed at the identification of the boundary zones of secondary structures, the estimation of the hazard level of deformation processes and the adoption of proactive measures.
\end{abstract}

\section{Introduction}

The modern society satisfies its needs in raw materials at the expense of open-cut and underground mining. And throughout the history of mining some of the constraints determining the mining efficiency and, at the same time, some of the threats to the safe mining are the intrinsic geomechanical processes and phenomena in the rock mass. First of all they include: rock pressure, rock movement, emissions of coal and gas, rock bumps and technogenic earthquakes appeared in the wake of rising the degree of technogenic impact on the rock mass during mining of solid minerals and especially of hydrocarbons. The founder of the Russian geomechanical science, I. M. Bakhurin (1946), said in the middle of the last century that "we'd be about right if we said that the entire history of mining, the entire history of finding the best research systems is a history of struggle with the rock

* Corresponding author: sashour@igd.uran.ru 
movements", understanding by rock movements at that period all the rock mass deformation processes accompanying the development of mineral deposits to whose research the geomechanical science is devoted [1].

The studies of geomechanical processes and phenomena made over the past nearly a century period have fully confirmed the correctness of those views. In fact, all the geomechanical processes and phenomena carrying a threat to the mining safety and impeding the mining efficiency as well as violating the safety and comfort of the surrounding population are connected, directly and sometimes indirectly, with deformation processes caused by interference in the natural environment. The comprehensive role of deformation processes in destabilization of the rocks in mines of different purposes and in generating the emergency situations has predetermined that the geomechanics studying the regularities of deformation processes in the rock mass under the conditions of its natural occurrence and in the fields of mining influence has the status of the key in ensuring the safe and efficient mining. Its practical use requires the knowledge of the basic factors involved in generating the emergency situations and the regularities of their interaction.

Until recently, in the mining science and practice of mining works the rock mass was considered and, in many cases, is being considered as a stable, equilibrium environment being in a homogeneous stress state generated by its own rock weight and tectonic forces arising from the geodynamical processes undermined in the past geological periods. The destabilization occurred only from technogenic mining activity. Such a simplified approach to the rock mass of the upper part of the lithosphere as an integral part of the Earth does not reflect its endogenous and exogenous processes and phenomena and leads to serious catastrophes with tragic consequences. Among them, the most large-scale catastrophes should include gas emissions and inflammations with mass mortality of people in coal mines, rock bumps and technogenic earthquakes, mine floods whose fresh examples have occurred in the past decades: Mine Sokolovskaya (SSGPO, 2005), mine Magnetitovaya (VGOK, 2009), mine Mir (ALROSA 2017). Along with such large-scale catastrophes, the development of mineral deposits is constantly accompanied with other smaller-scale emergency events. The negative consequences caused by discrepancy of projected processes and phenomena taken in the mining designs to feasible ones determine the relevance of the geomechanical studies over the years, growing as the mining production expands and the mining descends down to deeper depths.

The studies of the Ural school of geomechanics, established in the Institute of mining of Ural Branch of RAS have allowed on the basis of the confirmed data on the fundamental properties of the rock mass, such as the hierarchical blocking and constant mobility, to identify a number of basic regularities of processes and phenomena proceeding in the rock masses in their natural occurrence and in the reach of the technogenic mining activity. The studies have been conducted in mining enterprises with open-cut and underground developments of solid mineral deposits in the Urals and Kazakhstan as well as in the mineable hydrocarbon fields in Western Siberia and during the construction of the subway in Ekaterinburg [2].

\section{Methods of study}

The magnitude of the processes and phenomena proceeding in the mining and entering the circle of interests of the geomechanics leads to an integrated approach to the implementation of the research. Acquisition of baseline information is carried out by experimental methods under field conditions. Their following interpretation is performed with the use of the theoretical methods and the computer modeling using the apparatus of continuum mechanics. 
The research has established that the behavior and the state of rock mass, as in its natural state and in the area of mining activities, are determined by its stress-deformed state formed with two fundamental properties of the rock mass - its hierarchical block structure and recent geodynamical movements, supplying it with continuous mobility [3-9]. In accordance with this, the methods of research are focused primarily on the study of the structural features of rock mass, which is produced by geophysical methods of remote sensing [10,11]. The hierarchical block environment plays a determining role in the behavior of rock mass and the stability of mining objects, creating serious problems in the predictive assessment of the reliability and safety of mining structures of various sizes. So, for example, the preparatory mines require to estimate the behavior of the volumes of rock mass with the dimensions reckoned by the tens meters, and the open-pit side requires to estimate the behavior of rock mass with the dimensions of the hundreds of meters and sometimes kilometers. This task is solved with the electrometry methods in various versions, including the electrotomography, the method of georadar sensing and spectral profile shooting.

The stress-deformed state of rock mass in natural conditions is generated under its own rock weight and tectonic activity which is currently characterized by the recent geodynamical movements divided conditionally into trend and cyclical short-term movements. In the area of mining influence the natural factors are added by technogenic factor which disturbs the natural dynamic balance creating a secondary stress-deformed field around the mines depending on their parameters. The recent geodynamical movements are studied with the geodetic methods by monitoring the changing of coordinates of geodetic signs of observing stations on the earth's surface by the method of satellite positioning systems (GNSS). The measurements performed by the complex GNSS have showed its high efficiency to meet the challenges of the geomechanics, and its application enables not only the discrete measurements, but also the regular monitoring of deformations and stresses occurring in the earth's crust [12, 13]. The spatial coordinate binding of geodynamical polygons is performed by the double-system (GPS-GLONASS) and doublefrequency receivers of geodesic class whose potential and actual accuracy corresponds to 3$5 \mathrm{~mm}+1 \mathrm{ppm}$ in plan and $5-7 \mathrm{~mm}+1 \mathrm{ppm}$ in height.

During the first stage the current spatial coordinates of geodynamical polygons are determined, while the centering and orientation of geodesic constructions are carried out using the permanent GNSS stations (CORS) of the Ural region and the global network IGS with coordinate referencing to the International Terrestrial Reference Frame (ITRF) [14].

The trend movements of geodynamical polygons and observing station benchmarks are determined on the basis of the analysis of the spatial coordinate increments (vectors) $\Delta \mathrm{X}$, $\Delta \mathrm{Y}, \Delta \mathrm{Z}$ in the intervals between repeated measurement cycles, and the cyclic short-term movements are determined by the continuous measurements [15]. The interval deformations received as a result of instrumental measurements using the mathematical apparatus of continuum mechanics are converted into a vector and tensor representation of deformation field with the allocation of the main components of the deformation tensor [16].

During the second stage the program of instrumental observations includes the determination of high-altitude observing station benchmarks and soundings of courses between the benchmarks as well as the spatial coordinating using the complex of satellite geodesy and the special tasks for diagnostics of geodynamical activity of the investigated territory. As a result of instrumental observations in the vertical plane the benchmark subsidences and the dip deformations as well as the dip moveout velocities are determined; in the horizontal plane the movement vector parameters and the horizontal tensioncompression deformations as well as their velocities are determined. 
The results of instrumental measurement of movements and deformations are visualized by building a vector field of horizontal displacements (Figure 1) and tensors of horizontal deformations of regular geomechanical network model built by the Finite Element Method (FEM). The finite element network boundary and form change reflecting the investigating area deforming nature is also analyzed. The key elements of FEM model are points of the geodynamical polygon applications and the observing station benchmarks for which the spatial movements between performed repeated cycles of measurements have been determined.

\section{Research results}

The results for the monitoring of recent trend geodynamical movements in the open-pit mines of chrysotile asbestos for the period from 2006 to 2017 in the form of offset vectors are presented in Figure 1 «a». The total values of offsets caused by the recent geodynamical movements and by the changing of the open-pit mine parameters, as it appears from Figure, are different and vary in the different areas of the open-pit mines from $10-15$ to $60-70$ $\mathrm{mm}$. The directivity of offset vectors is also inhomogeneous. In many respects it is technogenic factor whereby the directivity inside the open-pit mine prevails, however the observed variation in their directions and values indicates a significant influence of the initial geodynamical movements.

The inhomogeneity and mosaic structure of stress-deformed state contrasts especially in the field of horizontal deformations submitted in Figure $1\langle b »$ in the form of deformation tensors obtained by the Finite Element Method (FEM) using the offset vectors as boundary conditions.

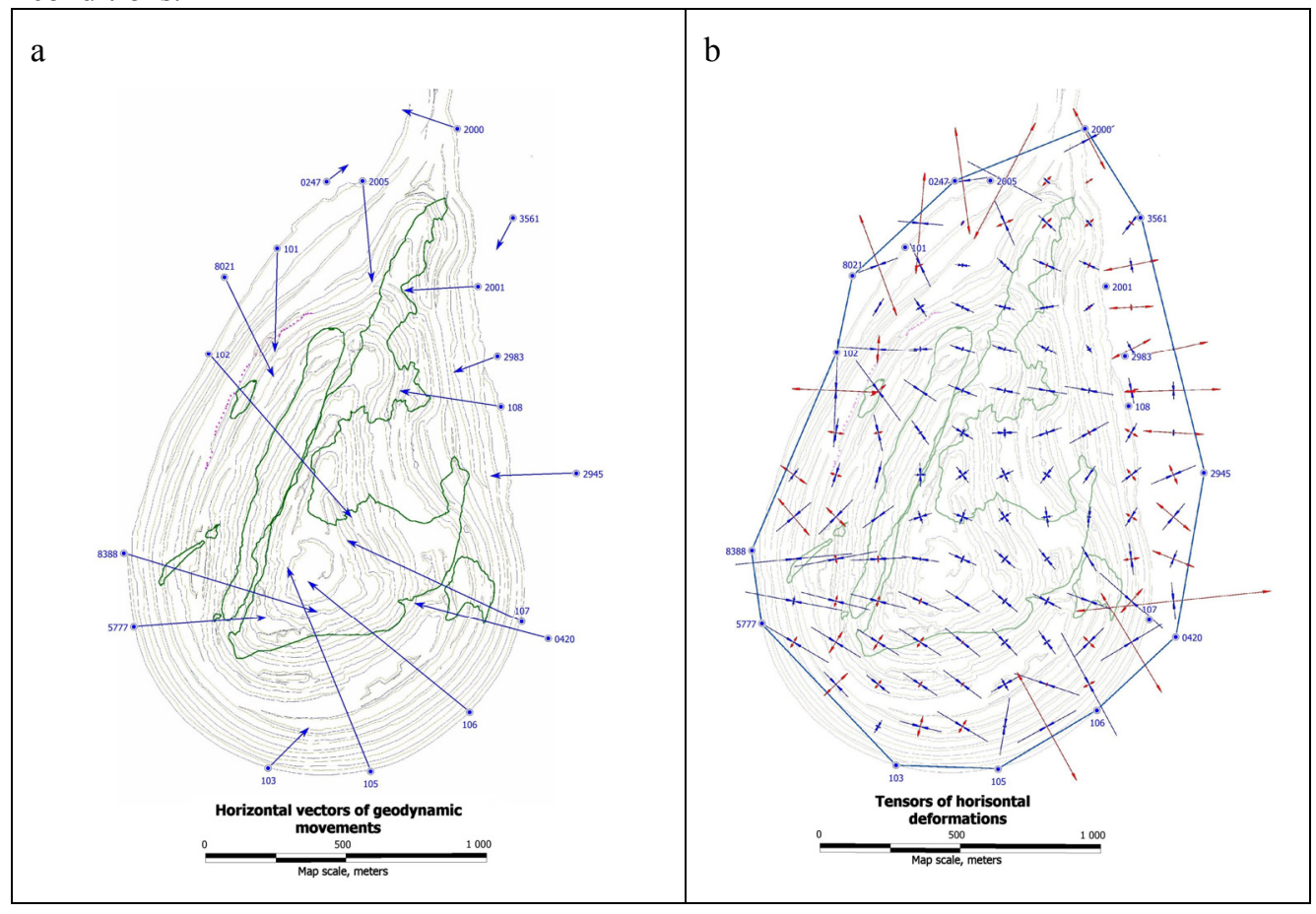

Fig. 1. Geodynamical movements and deformations in the open-pit mine area: a - Horizontal vectors of recent geodynamical movements in the open-pit mine area for the period from 2006 to 2017 ; b - Tensors of horizontal deformations caused by recent geodynamical movements in the openpit mine area at same time 
By the pit outline prevail the tensors with various signs of deformation - tension and compression differing in values. In the central part the tensors are more homogeneous and presented by the minor compressions in both main directions.

\section{Discussion of results}

The interpretation and analysis of the research results for the stress-deformed state at the various enterprises which develop the mineral deposits in open-cut and underground ways under various geomechanical conditions have revealed the basic regularities of the development of deformation processes in rock mass which play a key role in the safety and efficiency of mining. The research has established the following:

- - the hierarchical block environment of rock mass, being in constant mobility under the influence of trend and short-term cyclic recent geodynamical movements, is subjected to the secondary structuring;

- the consequence of secondary structuring of hierarchical block mass is a discrete mosaic structure of the stress-deformed state, and the recent geodynamical movements concentrate on the borders of secondary structures, causing the deformations which exceed intrablock and integral movements by 2-5 times (Figure 2);

- the mosaic structure of the stress-deformed state is amplified by interblock counter movements on the borders of secondary structures, creating the zones of concentration of compressive stresses and the zones of depression where rock mass can be completely freed of compressive stresses (Figure 3);

- abnormal deformations in the mosaic stress-deformed state caused by the secondary structuring and constant mobility are the main source of the hazardous impact on technogenic mining objects in the depression zones [17] (Figure 4) as well as in the areas of concentration of compressive deformations.

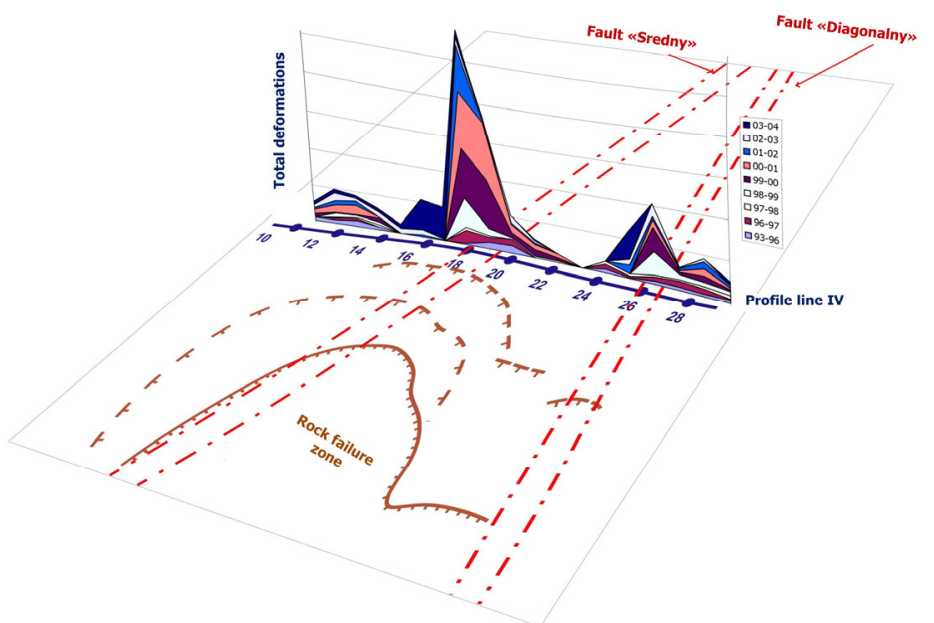

Fig. 2. Abnormal values of deformations on the borders of secondary structures

Thus, the performed research has identified the mechanism and regularities of the development of catastrophic deformation processes in rock mass, as reflected in the shear fractures caused by the mosaic structure of the stress-deformed state with a wide range of values of the components of the stress and deformation tensors in the hierarchical block environment under the influence of recent geodynamical movements, which establishes the key status to the geomechanics in ensuring the safety and efficiency of mining. The 
technology of the geological environment diagnostics for the forecast of hazardous mining conditions allows for the identification of boundary zones of secondary structures, the estimation of the hazard level of deformation processes and the adoption of proactive measures.

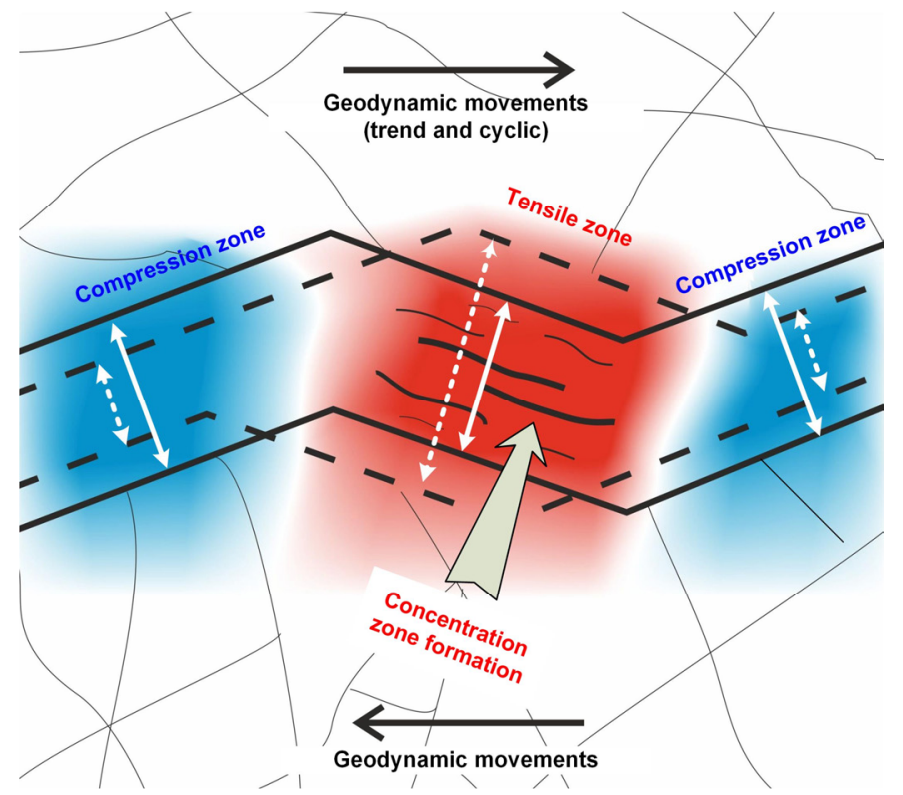

Fig. 3. Diagram of development of mosaic structure of the stress-deformed state under the influence of interblock movements of secondary structures

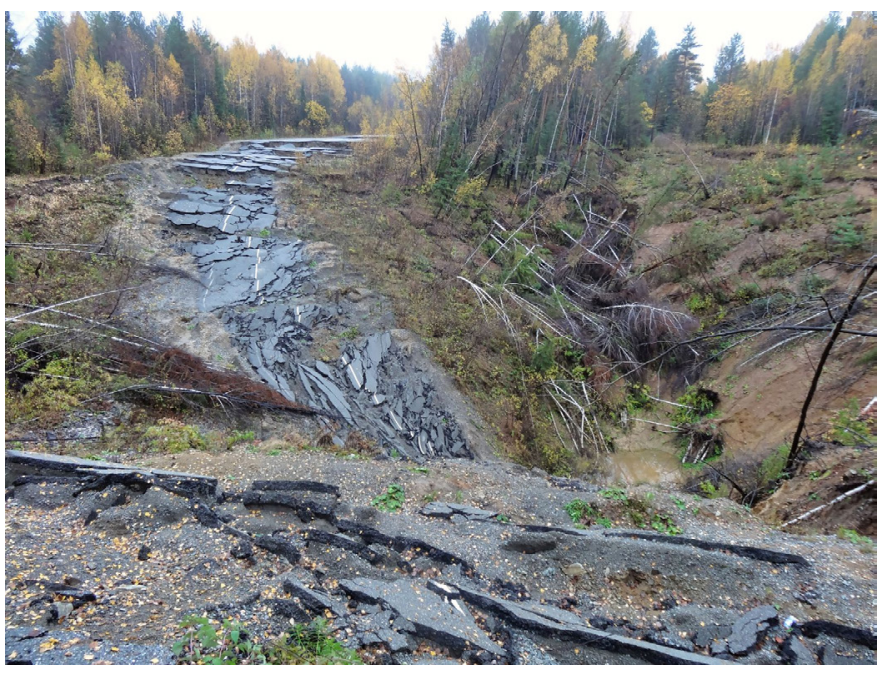

Fig. 4. Formation of the caving zone over the developed deposit in depression zone

\section{Conclusion}

The formation of the stress-deformed state of the upper part of the lithosphere during mining is determined by the fundamental properties of rock mass - hierarchical block structure and constant mobility under the influence of recent geodynamical movements, and 
their combination results in open non-linear system self-organizing in space and time under the influence of the phenomenon of secondary structure.

The phenomenon of secondary structure forms a mosaic structure of the stress-deformed state, determining the uneven impact of deformation processes in the mining objects.

The main source of emergency and catastrophic events at the mining objects are the interblock movements between secondary structures whose parameters exceed the intrablock and integral movements by $2-5$ times.

The key to ensuring the safety and efficiency of mining is diagnostics and predictive assessment of the impact of the geomechanical processes and phenomena on mining objects on the basis of identification of boundary zones of secondary structures and determination of the parameters of the movements and deformations in these zones, taking into account the ratio between the object sizes and the parameters of secondary structures.

\section{Thanks}

In the preparation of the paper the authors have used the results of the multi-year fundamental and applied research performed by a team of geomechanics of the Institute of mining of Ural Branch of RAS at the mining enterprises of the Ural Region and Kazakhstan, wherefore the author team expresses its deep appreciation to their colleagues as well as to engineers and technical workers of mining enterprises who took the liveliest participation in solving the geomechanical production problems

\section{References}

1. 1. I. M. Bakhurin, Rock Movement Under Influence of Mining (Gostopizdat, Moscow, 1946) 229 pp. [in Russian].

2. S. V. Usanov and Yu. P. Konovalov, Problemy nedropolzovaniya, 4 (11), 104 (2016). [Mining Problems, No. 4 (11), 104 (2016)]. DOI: 10.18454/2313-1586.2016.04.104

3. S. V. Usanov, Yu. P. Konovalov and O. D. Zheltysheva, Gorny zhurnal, 1, 36 (2012).

4. M. A. Sadovsky, "Natural Rock Lumpiness", Dokl. Akad. Nauk SSSR, 4, Vol. 247, 829 (1979).

5. M. A. Sadovsky, "Hierarchy from Dust to Planets". Earth and Universe. 6. 4 (1984).

6. Yu. O. Kuzmin, Modern Geodynamics and Estimation of Geodynamical Risk at Mining, (Agenstvo ecologicheskikh novostei, Moscow, 1999) 220 pp. [in Russian].

7. A. D. Sashourin. Mining Magazine, 1, 29 (2012); Eurasian Mining. 1, 17 (2012).

8. A. A. Panzhin. Mountain Information and Analytical Bulletin. 9, 313 (2010).

9. Yu. P. Konovalova. Mountain Information and Analytical Bulletin, 7, 269 (2010).

10. V. V. Melnik and A. L. Zamyatin. Mining Informational and Analytical Bulletin. 4, 226 (2005). Dokl. Akad. Nauk SSSR, No. 4, Vol. 247, 829 (2005) [Sov. Phys. Dokl., No. 4, Vol. 247, 829 (1979)].

11. V. V. Melnik. Mine Surveying and Mining, 6, 56. (2014).

12. Gao Jing-Xiang, Hu Hong. Procedia Earth and Planetary Science. 1(1). P. 1081-1088. (2009).

13. A. A. Panzhin, Mining Magazine, 1, 39 (2012).

14. A. A. Panzhin. Herald of the Magnitogorsk State Technical University named after G. I. Nosov, 1 (49), 22 (2015).

15. Fengshan Ma, Haijun Zhao, Yamin Zhang, Jie Guo, Aihua Wei et al. Journal of Rock Mechanics and Geotechnical Engineering. 4 (1). P. 82-87. (2012).

16. B. T. Mazurov, A. A. Panzhin, A. A. Silaeva. Geodesy and cartography. 3. 35 (2016).

17. V. I. Ruchkin. Mountain information and analytical bulletin. 5, 45 (2013). 